\title{
Selected papers from the international conference Academic Publishing in Europe: The Impact of Publishing
}

\author{
20-21 January 2009
}

The fourth APE - Academic Publishing in Europe - Conference in the Berlin Brandenburg Academy of Sciences, was about 'the impact of publishing'. The APE 2009 spectrum varied from usage and users, peer reviewing and quality assurance, branding, funding and investing, business models, new types of information, enabling technologies, search engines, to research excellence, dissemination, access and sharing of knowledge.

In this issue we present the APE 2009 Short Conference Report, written by Svenja Hagenhoff and Chris Armbruster, and we publish a relevant selection of papers from this conference. As there was simply too much material, we did not attempt to publish full proceedings this time.

Most presentations can be found under 'APE Literature' on the APE 2009 website: www.ape2009.eu.

APE Conferences encourage the debate about the future of scientific publications, information dissemination and access to scientific results. They offer an independent forum for 'open minds' with a free exchange of opinions and experiences between all stakeholders.

Participants were: academic, educational, scientific, technological, medical, legal and professional publishers, university presses, scientists, authors, editors, librarians, teachers, learned and professional societies and associations, funding agencies, politicians and policy makers, subscription agencies and booksellers, recruiting agencies and technology providers.

Academic Publishing and library activity face significant technical, financial and political challenges in the coming years. This may have major consequences for scholarly communication. Europe is the largest producer of scientific research and scholarly knowledge with a very strong publishing and library culture, with different scientific and professional cultures and a multitude of languages, but the acceptance, requirements and expectations are changing rapidly. Although the supportive role of academic organisations, societies and the private sector is subject to an intensive debate, there is a lot of uncertainty about current challenges and possibilities of the scholarly communication system, like new business models, the role of established players, funding schemes, quality and integrity of information, intellectual property, or life cycle and preservation of digital objects. Those issues are often discussed fiercely and emotionally, and there seems to be little interaction and knowledge exchange between the different players of the scholarly communication system, like librarians, publishing houses, university presses, scientists, funders and policy makers. 
The fifth APE Conference will be held 19-20 January 2010 in Berlin. We will focus on the roles of researchers, librarians and publishers in scholarly communication. The programme can be found on the website www.ape2010.eu.

Arnoud de Kemp

Editor of APE 2009 